\title{
CLUSTERING OF FIXATION TARGETS IN MULTIFIXATION CAMPIMETRY
}

\author{
ERKAN MUTLUKAN, BERTIL E. DAMATO and SHERINE M. TAVADIA \\ Glasgow
}

\begin{abstract}
SUMMARY
The multifixation campimeter has a central test stimulus and a series of numbered fixation targets and uses the patient's eye movements to position the stimulus in the visual field. The stimulus is constantly exposed so that the patient reads the numbers and identifies any which are associated with its disappearance. The aim of this study is to identify the effect of clustering fixation targets on sensitivity. Two hundred and seventy-two eyes of 139 normal individuals were tested with a multifixation campimeter in which either one or two of the fixation targets corresponded to the physiological blind spot. Sixty-nine individuals (138 eyes) were tested with chart $A$ and 70 individuals (134 eyes) with chart $B$. The second fixation target increased the blind spot detection rate from $65 \%$ to $85 \%$ of the eyes respectively. In $10 \%$ of eyes the blind spot was detected on only one of two examinations. The performance of multifixation campimetry is improved if fixation targets are clustered in vulnerable parts of the field and distributed so as to test the blind spot repeatedly during the examination. Inconsistent results are an indication for re-examination of selected points, with intermittent stimulus presentation.
\end{abstract}

Perimetry is still not widely available in the community because of its dependence on costly equipment and skilled personnel. In optometric practice, conventional perimetry is not performed routinely even if the equipment is available. ${ }^{1}$ The main limiting factor is the need for the patient to keep the eye immobile throughout the examination; this demands the supervision of a skilful examiner or a computerised monitoring system, both of which increase the cost of the examination. A technique for examining the visual field has recently been developed to overcome this obstacle by using the patient's eye movements to position an inert test stimulus in the visual field (i.e. oculokinetic perimetry, OKP). ${ }^{2}$ The examination is performed with a 'multifixation campimeter', which consists of a tangent

Correspondence to: Dr. Erkan Mutlukan, Tennent Institute of Ophthalmology, University of Glasgow, 38 Church Street, Glasgow G11 6NT, UK. screen with a central fixed test stimulus and a series of eccentric fixation targets; as the patient looks at each fixation target in turn, from the correct distance, the stimulus is precisely positioned at known locations in the visual field.

The examination of a large number of points throughout the visual field in all patients would be ideal, in theory, but is impracticable because such a strategy would be too time-consuming and exhausting for the patient. When screening for disease, it is therefore necessary to examine a small number of points which are carefully selected according to known patterns of visual field loss in glaucoma and other important diseases. ${ }^{3}$ If speed and economy can be achieved without sacrificing sensitivity and specificity, then visual field examination might become routine in the community, where it could be administered in combination with other types of examination for the detection of disease.

As with conventional methods, multifixation campimetry should ideally be performed with intermittent exposure of the test stimulus, so that the patient says when the stimulus appears and disappears. It has been found that most individuals can reliably detect visual field defects even when the stimulus is constantly exposed, so that it is possible to perform the examination more rapidly. ${ }^{4}$ There are some patients, however, who tend to rush the examination, which increases the probability of missing small defects. For this reason a multifixation campimeter for the screening of glaucoma has additional fixation targets designed to slow the eye movements when the stimulus is located in the most vulnerable parts of the visual field.

In this study we investigated the effect of clustering fixation targets on sensitivity, using the normal blind spot.

\section{MATERIALS AND METHODS}

Subjects with a visual acuity of 6/12 or better and without any known ocular or systemic disease were selected randomly from hospital personnel and persons escorting patients to our hospital clinic. Two hundred and seventytwo eyes (136 right, 136 left) of 139 perimetrically inex- 
perienced individuals (66 males, 73 females; age range 21-78 years, mean 45 years) were included in the study.

Two different charts were used (i.e. chart A and chart B), which had either one or two fixation targets corresponding to the physiological blind spot (Fig. 1). Sixtynine individuals were tested with chart $A$ and 70 with chart B. All patients were examined with a black $1.5 / 400 \mathrm{~mm}$ stimulus on a white background.

The multifixation campimetry was performed under normal lighting conditions (150 lux). The right eye was tested first. Presbyopic and ametropic individuals were fitted with an appropriate optical correction. The same examination protocol was followed in each patient. The subject was asked to look at the first fixation target (i.e. number ' 1 ') and to say whether or not the stimulus was visible. Next, the subject was asked to read each number aloud and to report any numbers that were associated with disappearance of the stimulus. The results were recorded by the examiner without comment so as not to cause any bias. After examining the fellow eye, both eyes were examined a second time. If the test stimulus disappeared
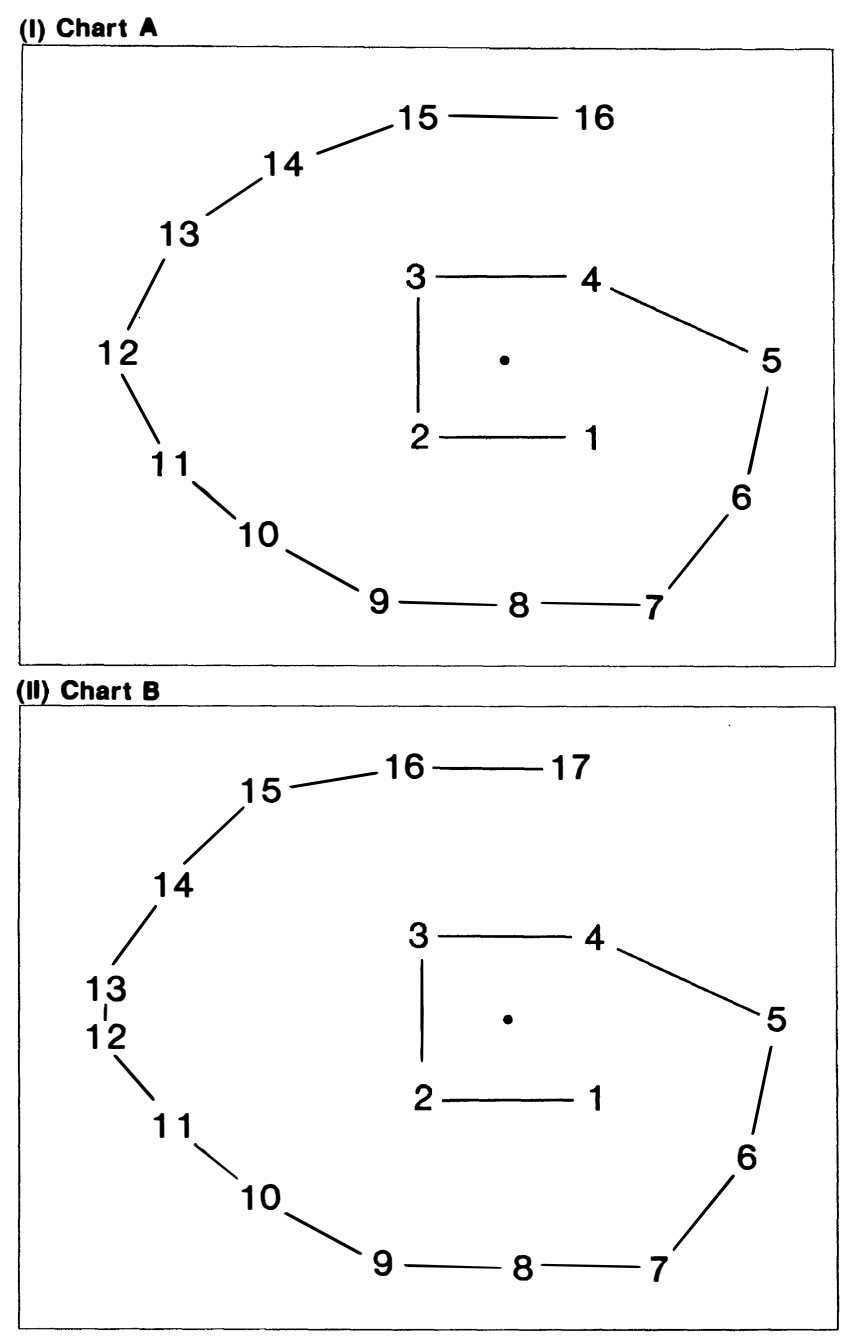

Fig. 1. Multifixation campimeters. Chart A has a single fixation target (i.e. number '12') corresponding to the physiological blind spot. Chart B has fixation targets '12' and '13' corresponding to the blind spot. (Charts shown are for right eye only.) on both examinations when the patient looked at the number or numbers corresponding to the blind spot, the result was regarded as 'non-fluctuating positive'. If the blind spot was detected during one of the two consecutive examinations the result was categorised as 'fluctuating positive'. If the blind spot was not detected on either examination the result was categorised as 'false negative'.

\section{RESULTS}

With chart A, which had one fixation target corresponding to the blind spot, a non-fluctuating positive result was obtained in $75 / 138$ eyes $(54 \%)$; a fluctuating positive result occurred with $14 / 138$ eyes (10\%); and a false negative result occurred with $49 / 138(36 \%)$ (Table I).

With chart B, which had two fixation targets corresponding to the blind spot, a non-fluctuating positive result was achieved in a total of 102/134 eyes (76\%); with one or both fixation targets in 20 and 82 eyes respectively. With $12 / 134(9 \%)$ a fluctuating positive result only was obtained. A false negative result occurred with 20/134 (15\%).

The increase in the non-fluctuating positive result rate with chart B as compared with chart A (i.e. $76 \%$ vs. $54 \%$ ) was statistically significant in both right and left eye groups $(t$-test, $p<0.01)($ Fig. 2$)$. The decrease in the false negative result rate (i.e. $15 \%$ vs. $36 \%$ ) with clustering of the fixation targets on chart B was also statistically significant $(p<0.01)$. No significant difference was found between the fluctuating positive result rates of charts $A$ and $\mathrm{B}(p<0.5)$.

\section{DISCUSSION}

Conventionally, a scotoma is regarded as clinically significant if it is at least $3^{\circ}$ in diameter. ${ }^{3}$ The physiological blind spot, with constant diameters of $5.5^{\circ}$ by $7.5^{\circ}$, was ideally suited for the purposes of this study.

Each subject was examined with either chart A or chart $\mathrm{B}$, but not both, so as to avoid bias from a learning effect.

The addition of a second fixation target increased the non-fluctuating positivity rate from $54 \%$ to $76 \%$. This finding suggest that, with multifixation campimetry, the greater the number of points examined within a scotoma

Table I. Detection of physiological blind spot according to the number of corresponding test stimuli

\begin{tabular}{lrrr}
\hline & \multicolumn{3}{c}{ Eye } \\
\cline { 2 - 4 } & \multicolumn{1}{c}{ Right } & Left & Both \\
\hline $\begin{array}{l}\text { Non-fluctuating } \\
\text { Chart A }\end{array}$ & $37(53.6 \%)$ & $38(55.1 \%)$ & $75(54.3 \%)$ \\
Chart B & $54(80.6 \%)$ & $48(71.6 \%)$ & $102(76.1 \%)$ \\
Fluctuating positive & & & \\
Chart A & $5(7.2 \%)$ & $9(13.0 \%)$ & $14(10.1 \%)$ \\
Chart B & $5(7.5 \%)$ & $7(10.5 \%)$ & $12(9.0 \%)$ \\
False negative & & & \\
Chart A & $27(39.1 \%)$ & $22(31.9 \%)$ & $49(35.5 \%)$ \\
Chart B & $8(11.9 \%)$ & $12(17.9 \%)$ & $20(14.9 \%)$ \\
Total & & & \\
Chart A & $69(100 \%)$ & $69(100 \%)$ & $138(100 \%)$ \\
Chart B & $67(100 \%)$ & $67(100 \%)$ & $134(100 \%)$ \\
\hline
\end{tabular}




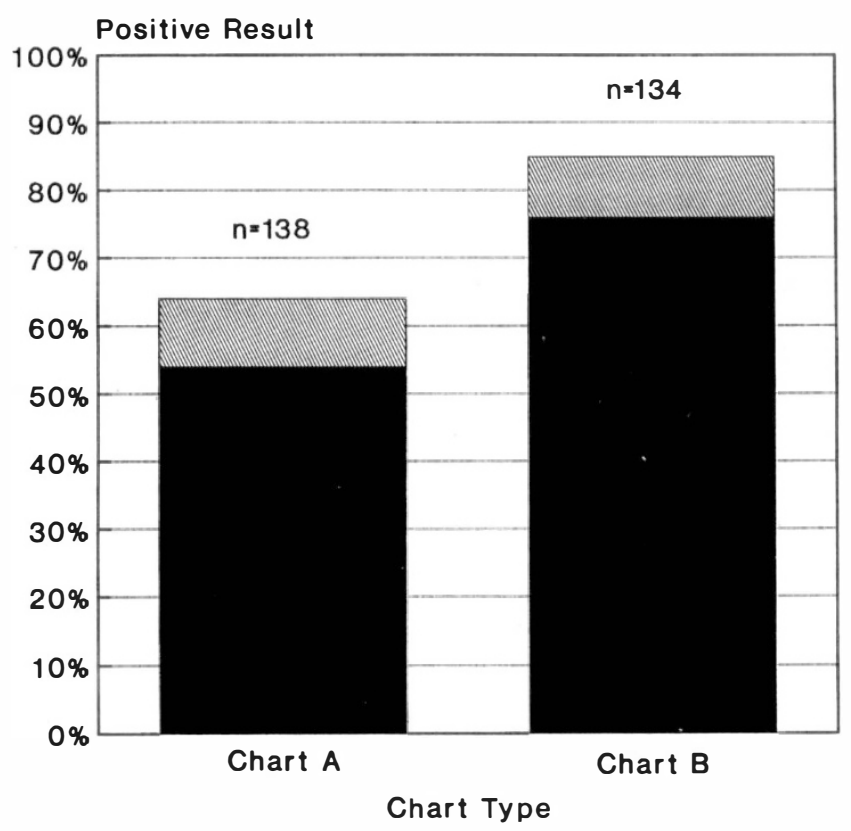

Positive Result

Non-Fluctuating $\quad \mathbb{\quad}$ mele fluctuating

Fig. 2. Repeatable (non-fluctuating) and non-repeatable (fluctuating) misses in the blind spot using charts $A$ and $B$.

he greater is the probability of detecting the defect. The clustering of fixation targets in vulnerable parts of the visual field is therefore useful.

At present, a test result is considered positive only if the stimulus consistently disappears when the examination is repeated. The results of this study suggest that, with small defects only, such a strategy reduces the sensitivity of an examination by approximately $10 \%$. When an inconsistent or fluctuating positive response occurs, the result should be regarded as inconclusive and selected points reexamined with an intermittently presented stimulus. In other words, the patient should look continuously at a number and say when the stimulus appears and disappears, while the examiner uncovers and covers the stimulus with a white card.

In view of the results of this study, future multifixation campimeters should be designed so as to test and re-test the blind spot several times during the visual field examination as in conventional perimetry. ${ }^{5}$

In conclusion, this study confirms the value of clustering fixation targets, suggests an alternative method of interpreting inconsistent results, and identifies the need for repeated testing of the blind spot during the examination.

We gratefully acknowledge the support of the International Glaucoma Association and the Royal National Institute for the Blind.

\section{REFERENCES}

1. Tuck M, Crick R. Testing and referral for chronic glaucoma. Health Trends, 1989;21:131-4.

2. Damato BE. Oculo-kinetic perimetry: a simple visual field test for use in the community. Br J Ophthalmol 1985;69: 927-31.

3. Anderson DR. Perimetry with and without automation. 2nd ed. St Louis: Mosby, 1987.

4. Damato BE, Chayla J, McClure E, Jay JL, Allan D. A handheld OKP chart for the screening of glaucoma: preliminary evaluation. Eye 1990;4:632-7.

5. Heijl A, Krakau CET. An automatic static perimeter: design and pilot study. Acta Ophthalmol 1975;53:293-7. 